\title{
Modelos de velocidad de operación de carreteras rurales en terreno llano en Costa Rica
}

\section{(Speed profile prediction model of rural roads in flat terrain in Costa Rica)}

\author{
Domingo E. Delgado Martínez', Liosber Medina García², José M. Ulate Zárate³, \\ René A. García Depestre ${ }^{4}$
}

\begin{abstract}
Resumen
Por varias décadas, en la práctica ingenieril se ha evaluado la influencia del trazado de la carretera en la seguridad vial mediante el análisis de la consistencia; para ello es necesario contar con los perfiles de velocidad de operación del proyecto nuevo o de la carretera existente. Con este fin, generalmente, se emplean modelos de velocidad de operación existentes que fueron desarrollados para otras condiciones, regiones o países. En los análisis de consistencia del trazado es imprescindible contar con la información de la planta, el perfil del proyecto y la velocidad de operación de los vehículos. Una de las principales dificultades y fuentes de error en estos análisis radica en determinar la velocidad de operación. El objetivo del trabajo es desarrollar modelos de predicción de velocidad de operación para Costa Rica que consideren las características del trazado y las condiciones del parque vehicular. El procedimiento seguido incluye una serie de etapas, desde el análisis de las carreteras del territorio hasta el desarrollo de los modelos de predicción del perfil de velocidad y su validación. Como resultado de la investigación se proponen, por primera vez en Costa Rica, modelos de predicción de las velocidades de operación de las rutas nacionales con características rurales en terreno llano. Se demuestra que las ecuaciones propuestas presentan menores errores de estimación si se comparan con algunos de los modelos más usados internacionalmente.
\end{abstract}

\section{Palabras clave}

Consistencia del trazado, modelo de predicción, velocidad de operación, seguridad vial, carreteras rurales, terreno llano.

\begin{abstract}
For some time, a commonly used engineering practice has been to evaluate the influence of the geometric design of roads on road safety by analyzing geometric design consistency. When evaluating geometric design consistency, it is necessary to know the operating speed profiles of the road to be analyzed. To this end, a variety of models of operating speed profiles are used, which are designed for the specific conditions of a particular region or country. For any analysis of highway geometric design, it is important to have information related to the horizontal and vertical layout of the project, as well as to the operating speed of vehicles. However, one limitation, which is often a source of error, is determining the operating speed of the vehicles. The aim of this paper is to develop models for predicting vehicle operating speed in Costa Rica by considering the characteristics of the road geometric design and of the existing vehicles. The procedure followed in the model included a series of steps, including from the analysis of the roads in the region to the development of models for predicting the speed profiles, as well as its validation. For the first time, different models are proposed for rural national routes in flat terrain in Costa Rica. The study demonstrates that the proposed equations provide for lower estimations errors, if compared to international models.
\end{abstract}

\section{Keywords}

Geometric design consistency, prediction model, operating speed, road safety, rural roads; flat terrain

\footnotetext{
1 Universidad Latina de Costa Rica, San José, Costa Rica [domingo.delgado@ulatina.cr, https://orcid.org/0000-0002-16893769].

2 Universidade Federal de Goiás, Goiania, Brasil [Imedina@ufg.br, https://orcid.org/0000-0002-4091-3491].

3 Universidad Latina de Costa Rica, San José, Costa Rica [julatez@cfia.or.cr, https://orcid.org/0000-0002-7361-1449].

4 Universidad Central "Marta Abreu" de las Villas, Santa Clara, Cuba [renegdauclv.edu.cu, https://orcid.org/0000-00031957-4760].
} 


\section{Introducción}

En los últimos años, en muchos países se ha evaluado la seguridad vial a partir del análisis de la consistencia del trazado (Abebe \& Belayneh, 2018; Almeida et al., 2018; Echaveguren et al., 2020; Fitzpatrick et al., 2000; García et al., 2012; Kiran et al., 2017; Li et al., 2017; Llopis-Castelló et al., 2020; Llopis-Castelló et al., 2018; Xu, Lin, \& Shao, 2017). El análisis de la consistencia del trazado permite verificar si las condiciones geométricas de la carretera generan pequeños diferenciales de velocidad entre elementos consecutivos, ya sea curvas y rectas en horizontal o acuerdos, tanto cóncavos como convexos. La consistencia del trazado se define como la relación entre las características geométricas del trazado de la carretera y las expectativas del conductor (Leisch \& Leisch, 1977). Si hay correspondencia entre estos dos aspectos, la conducción del vehículo puede hacerse de modo continuo, sin sobresaltos, lo que incide favorablemente sobre la seguridad en la circulación. Aunque existen diferentes métodos para estimar la consistencia, los más utilizados han sido aquellos basados en el análisis de la velocidad de operación de los vehículos. Entre las principales ventajas de utilizar este tipo de análisis se destaca la posibilidad de aplicarlo tanto a proyectos nuevos como existentes.

García-Ramírez et al. (2017) y Goralzik y Vollrath (2017) explican que las causas o fuentes de error en la estimación de la velocidad de operación es una cuestión de gran complejidad debido a los factores de los cuales depende, tales como las características del conductor, los vehículos, el entorno, la geometría de la carretera y su operación y control. Otra de las posibles fuentes de variación en la velocidad de operación es la recolección de datos.

Investigadores como Wilches et al. (2020), Xu, Lin y Shao (2017) y Xu, Lin, Wang, et al. (2017) destacan la importancia del terreno y el diseño geométrico del trazado para obtener los modelos de perfil de velocidad. Otros como Echaveguren et al. (2020) resaltan la complejidad geométrica del trazado como un factor determinante en la variación de la velocidad de operación.

De igual forma, la rapidez con que ha aumentado la flotilla vehicular, en Costa Rica, es una cuestión de gran interés, debido a su efecto en la seguridad vial. En la

Figura 1 se muestran los datos históricos del crecimiento vehicular hasta el año 2017. La tasa de crecimiento del año 2017 respecto al 2016 era de un $5.4 \%$. La baja en la tendencia de crecimiento en 2009 corresponde con la recesión económica de 2008 y 2009.

A partir de la interpretación de los reportes de accidentes del tránsito disponibles en el Consejo de Seguridad Vial (COSEVI), para el período de 2015 a 2019 (COSEVI, 2020), se logra reinterpretar las causas de estos accidentes y crear un modelo de interrelaciones entre los principales factores que intervienen en los accidentes del tránsito. En la se muestra el modelo de los porcentajes de accidentes de tránsito según sus causas en el intervalo de 2015 a 2019. En el grafico se constata que en Costa Rica el factor humano es la causa fundamental de los accidentes; sin embargo, una parte no menos importante de estos accidentes es atribuible a las condiciones geométricas del trazado. Además, puede inferirse que un gran número de accidentes está relacionado con las interrelaciones de las condiciones geométricas del trazado con el factor humano, lo que por sí solo justifica la importancia del estudio. 
Figura 1. Crecimiento histórico del parque vehicular en Costa Rica

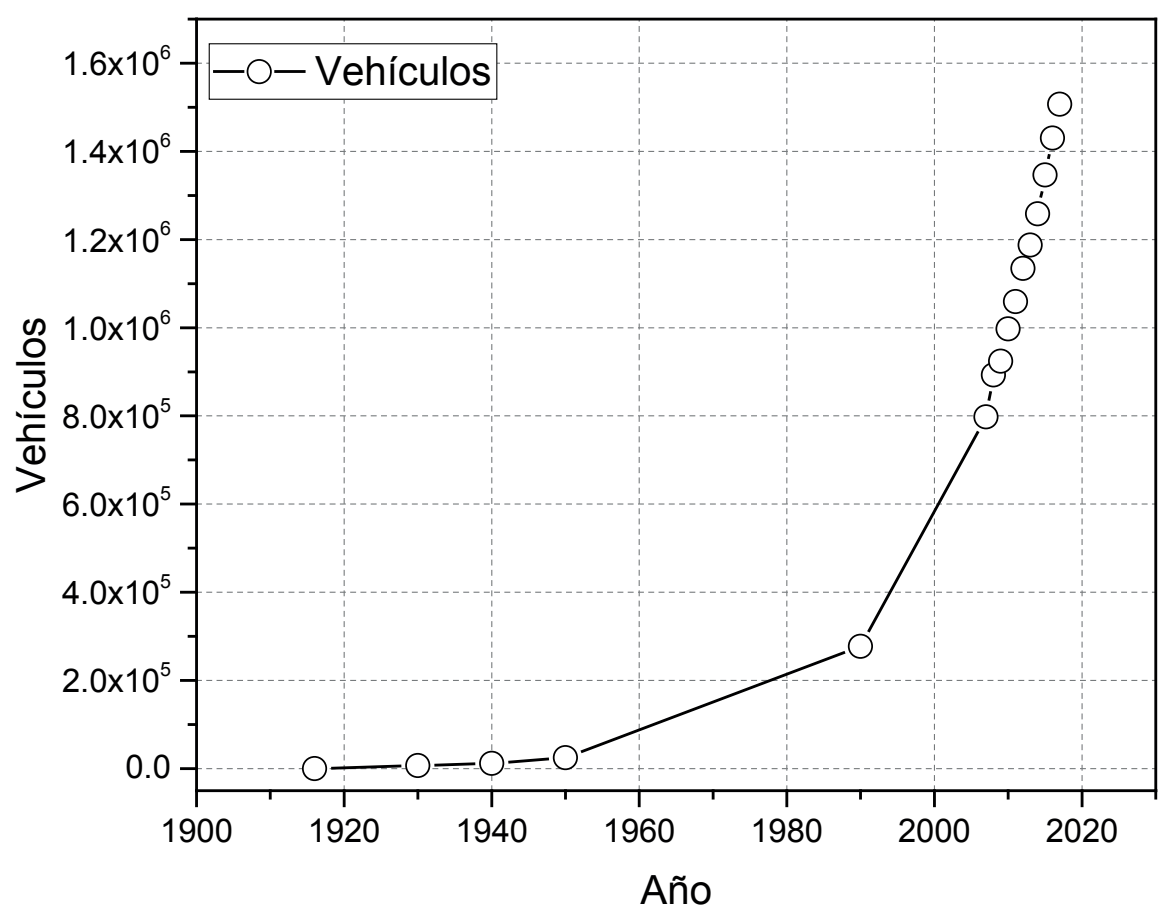

Nota: Tomado de COSEVI (2020)

Figura 2. Relación entre los elementos que intervienen en la accidentalidad (2015 a 2019)

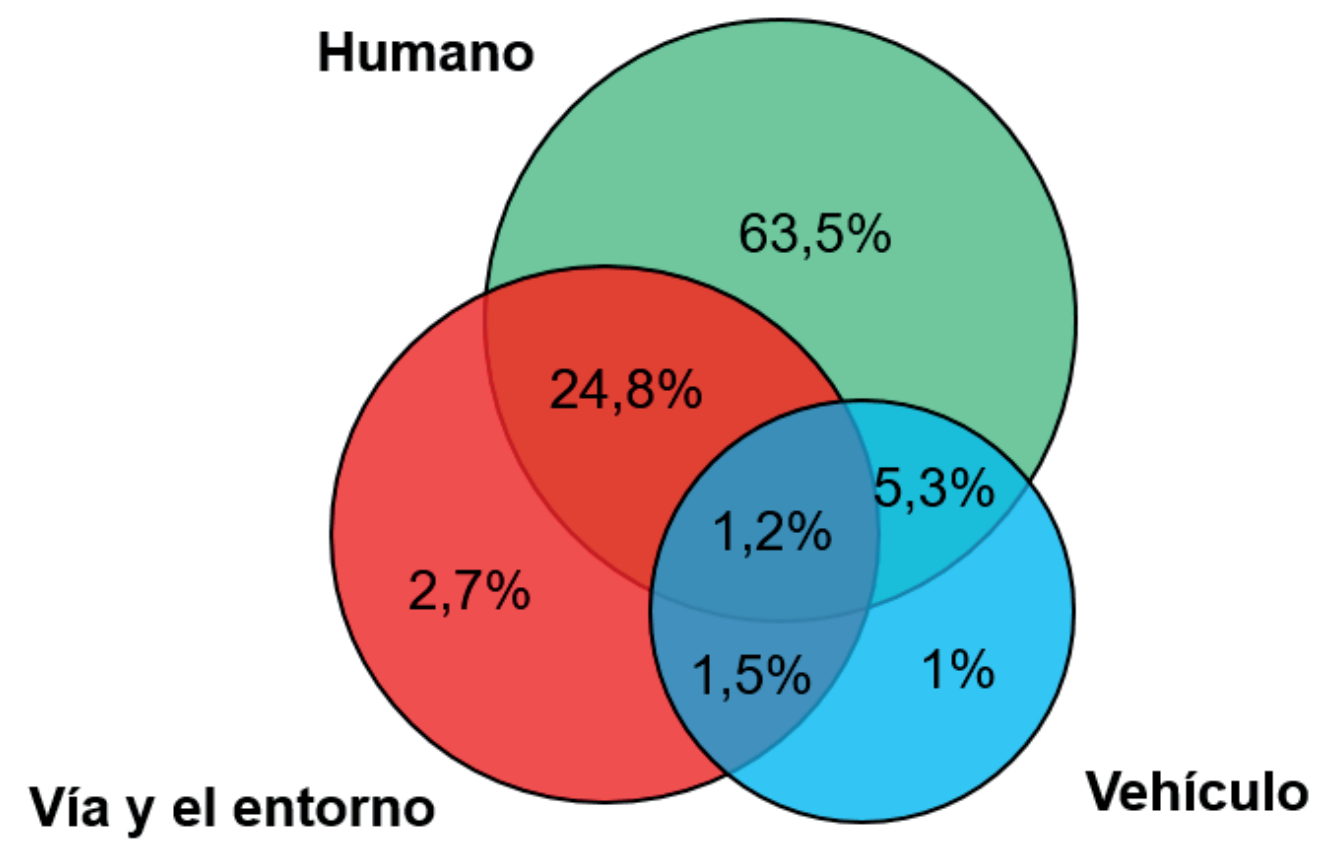


Varios investigadores coinciden en que la velocidad de operación se define como el percentil 85 de la distribución de velocidades a la que operan los vehículos ligeros en condiciones de flujo libre (Choudhari \& Maji, 2019; García-Ramírez \& Damián Alverca, 2019; Llopis-Castelló et al., 2019; Llopis-Castelló et al., 2018; Maji et al., 2018; Malaghan et al., 2020; Saleem \& Persaud, 2017; Sil et al., 2018). El percentil 85 es el más empleado porque representa aproximadamente la velocidad a la que operan los conductores y que se considera segura.

A lo largo de los años, se han propuesto varios modelos de velocidad de operación y uno de los más reconocidos es el desarrollado por Fitzpatrick et al. (2000). Los modelos de previsión de velocidad deben ser desarrollados o calibrados considerando las condiciones locales y, además, deben estar sujetos a revisiones a lo largo del tiempo. Las ecuaciones obtenidas para una condición no deben ser extrapoladas sin análisis previos, por lo que las condiciones locales deben analizarse de manera particular. Por lo tanto, cada país o región debe desarrollar perfiles de velocidad propios, que tomen en cuenta las características del parque vehicular y las condiciones geométricas del trazado.

La mayoría de las metodologías de evaluación de la consistencia del trazado han desarrollado perfiles de velocidad o ecuaciones de pronósticos de este parámetro (Fitzpatrick et al., 2000; Llopis-Castelló, et al., 2018; Sil et al., 2019).

En Centroamérica no se reportan trabajos donde se hagan estudios de velocidad de operación. En aras de implementar los estudios de consistencia del trazado en la evaluación de la seguridad vial en Costa Rica, es necesario desarrollar modelos propios de estos perfiles, que consideren las características del trazado y las condiciones del parque vehicular del país.

Teniendo en cuenta la importancia de considerar la influencia de las condiciones geométricas del trazado en la accidentalidad en Costa Rica, es fundamental desarrollar modelos de velocidad de operación que constituyan la base para llevar a cabo los análisis de consistencias del trazado y que tomen en cuenta las condiciones específicas del país, es decir, las características del parque vehicular y el trazado geométrico.

\section{Materiales y métodos}

A partir de la experiencia de varios investigadores, entre los que se destacan Fitzpatrick et al. (2000), y tomando como base fundamental experiencias propias García et al. (2012), se discuten algunos de los elementos más importantes en aras de establecer un procedimiento metodológico para el desarrollo de perfiles de velocidad propios aplicable al caso de Costa Rica.

Para establecer los perfiles de velocidad, es indispensable disponer de la información y los datos de los proyectos o carreteras ya existentes de la región objeto de estudio. Entre los parámetros más relevantes se encuentran la clasificación y el tipo de vía, el número de carriles, el tipo de terreno en que se encuentran, así como las características geométricas y la velocidad de operación. Los modelos de perfiles de velocidad se determinan en función de las múltiple combinaciones de trazado en planta y perfil, dependiendo de la categoría de la vía, tipo de terreno y criterios de diseño que establece el Manual centroamericano de normas para el diseño geométrico de carreteras (SIECA, 2011).

El procedimiento metodológico propuesto para determinar los modelos de velocidad de operación consta de las siguientes etapas: análisis de las carreteras del territorio y definición de parámetros fijos por investigar, determinación de los sitios y selección de la muestra, mediciones de velocidad en los sitios seleccionados, desarrollo de los modelos de predicción del perfil 
de velocidad y la validación de los modelos desarrollados. A continuación, se presenta una breve descripción de cada una de ellas.

\section{Análisis de las carreteras del territorio y definición de parámetros fijos por investigar}

Para desarrollar los perfiles de velocidad, es preciso contar con una caracterización de la red vial nacional del país o región que se desea evaluar. A partir de la disponibilidad de los datos de esta red vial, es que se debe establecer tipo de carretera para el cual se desarrollarán los modelos. En el caso de Costa Rica, la red nacional se clasifica en dos grupos: la Red Vial Nacional, administrada por el Ministerio de Obras Públicas y Transporte (MOPT), y la Red Vial Cantonal, administrada por las municipalidades correspondientes. La Red Vial Nacional cuenta con $5127 \mathrm{~km}$ asfaltados y 2594 km en lastre, para un total de 7721 km; la Red Vial Cantonal se compone de $5073 \mathrm{~km}$ asfaltados, $29917 \mathrm{~km}$ en lastre y $5194 \mathrm{~km}$ no clasificados, para un total de $40184 \mathrm{~km}$ (COSEVI, 2020). Con estos datos, Costa Rica presenta una densidad de carreteras de aproximadamente $0.79 \mathrm{~km} / \mathrm{km}^{2}$.

Otro aspecto importante que se debe tener en cuenta son los parámetros geométricos fijos y sus combinaciones. Los parámetros fijos de las carreteras son elementos que no varían a lo largo de su vida útil, siempre que no se realicen intervenciones. Los parámetros más significativos, con respecto a las características geométricas, son la pendiente y el radio de curvatura horizontal, grado de curvatura, longitud de curva y Kv.

\section{Determinación de los sitios y selección de la muestra}

Se denomina 'sitio' a la agrupación de una o varias curvas de características geométricas similares de la misma condición, ya sea curva horizontal, vertical, o su combinación. Para organizar el procedimiento de selección del sitio, se recomienda que las alineaciones, horizontal y vertical, se dividan en grupos, o que incluyan las combinaciones deseadas Fitzpatrick et al. (2000). Los estudios anteriores de García et al. (2012) y Fitzpatrick et al. (2000) reportan que para desarrollar perfiles de velocidad de operación se han de agrupar los elementos de características geométricas similares.

Cada uno de los tramos de la red vial se clasifica según las pendientes de la rasante y el terreno; de esta manera, se define a qué tipo de terreno pertenecen los sitios clasificados en cada condición o caso, ya sea llano ondulado o montañoso. Esta clasificación para el caso de Costa Rica se realiza con base en el manual de SIECA (2011).

En el estudio desarrollado por Fitzpatrick et al. (2000) se determinan diez combinaciones de sitios y, para cada uno de ellos, se establecieron los modelos de predicción de velocidad. En el caso del estudio de García et al. (2012), se definieron ocho tipos de sitios, lo que demuestra que estas combinaciones pueden variar en función de las condiciones locales, regionales o del país donde se desarrolle este tipo de estudio.

Una vez definida la localidad, región o país y tipo de terreno para el cual se establecerá el perfil de velocidad, se define la población con que se cuenta para el estudio y, a partir de ella, se determina una muestra representativa. El cálculo del número de muestras se hace utilizando la ecuación 1, que es recomendable utilizar cuando se conoce el tamaño de la población. 


$$
n=\frac{Z^{2} p q N}{N E^{2}+Z^{2} p q}
$$

Donde:

n tamaño de la muestra

$Z$ nivel de confianza

$p$ variabilidad positiva

$q$ variabilidad negativa

$N$ tamaño de la población

E precisión o error

\section{Mediciones de velocidad en los sitios seleccionados}

La velocidad de operación se determina para vehículos ligeros, en horario diurno, en condiciones climáticas favorables y con pavimento seco y en buen estado de conservación. Los estudios de velocidad deben ser elaborados en el punto de la curva donde se propicia un aumento de la velocidad de operación y se pueden utilizar diferentes técnicas, entre las que se destaca la utilización de la pistola láser.

Los estudios Fitzpatrick et al. (2000) y García et al. (2012) refieren que, para las condiciones que involucran rectas en curvas verticales, ya sean cóncavas o convexas, las velocidades deben ser medidas en el punto medio, exactamente donde se forma la curva vertical. En el caso de combinación de curvas horizontales con curvas verticales, las velocidades deben ser medidas en el punto medio de la curva horizontal, que también debe coincidir, desde la concepción del proyecto, con el punto donde se da el cambio de pendiente que genera el acuerdo.

Una de las expresiones estadísticas que puede ser utilizada para establecer el tamaño de la muestra de mediciones de velocidad es la ecuación 2, recomendada por Robertson et al. (1994).

$$
n=\frac{S^{2} K^{2}\left(2+U^{2}\right)}{2 E^{2}}
$$

Donde:

S Desviación estándar estimada $(\mathrm{km} / \mathrm{h})$

K Constante asociada al nivel de confianza deseado

U Constante asociada al percentil analizado

E Error permitido $(\mathrm{km} / \mathrm{h})$

n Tamaño de la muestra (número mínimo de mediciones de velocidad). 
Los valores de la desviación estándar estimada $S$ pueden ser obtenidos según recomendaciones de Robertson et al. (1994). En la Tabla 1 se presentan los valores que se sugieren para estos casos.

Tabla 1. Desviación estándar para determinar el tamaño de muestra

\begin{tabular}{|c|c|c|}
\hline Área de tráfico & Tipo de vía & Desviación estándar $(\mathbf{k m} / \mathbf{h})$ \\
\hline \multirow{2}{*}{ Rural } & Un carril & 8.5 \\
\cline { 2 - 3 } & Dos carriles & 6.8 \\
\hline \multirow{2}{*}{ Intermedia } & Un carril & 8.5 \\
\cline { 2 - 3 } & Dos carriles & 8.5 \\
\hline \multirow{2}{*}{ Urbana } & Un carril & 7.7 \\
\cline { 2 - 3 } & Dos carriles & 7.9 \\
\hline & Valor Recomendado & 8.0 \\
\hline
\end{tabular}

Nota: Tomado de Robertson et al. (1994)

De igual forma, para obtener los valores de la constante $K$, Robertson et al. (1994) recomiendan utilizar los valores de la Tabla 2.

Tabla 2. Valores de constante $\mathrm{K}$

\begin{tabular}{|c|c|}
\hline Nivel de confianza (\%) & Constante K \\
\hline 68.30 & 1.00 \\
\hline 86.60 & 1.50 \\
\hline 90.00 & 1.64 \\
\hline 95.00 & 1.96 \\
\hline 95.50 & 2.00 \\
\hline 98.80 & 2.50 \\
\hline 99.00 & 2.58 \\
\hline 99.70 & 3.00 \\
\hline
\end{tabular}

Nota: Tomado de Robertson et al. (1994)

Los autores Robertson et al. (1994) establecen los valores de la desviación estándar estimada $S$ en función del percentil de velocidad (ver Tabla 3).

Tabla 3. Valores de constante U

\begin{tabular}{|c|c|}
\hline Percentil de velocidad & Constante U \\
\hline 50.00 & 0.00 \\
\hline 85.00 & 1.04 \\
\hline 93.00 & 1.48 \\
\hline 95.00 & 1.64 \\
\hline
\end{tabular}

Nota: Tomado de Robertson et al. (1994) 
En caso de que las bases de datos, los proyectos que estén a disposición de los investigadores, entre otros, sean insuficientes para garantizar la representatividad del muestreo, lo más recomendable es recopilar la información complementaria con trabajos de campos, siempre y cuando existan sitios para ampliar la muestra. Estos estudios complementarios in situ facilitan la verificación o determinación de información para satisfacer el tamaño de muestra requerido para el trabajo.

\section{Desarrollo de los modelos de predicción del perfil de velocidad}

Al concluir las mediciones de velocidad en los sitios seleccionados, es necesario hacer un procesamiento de las estas. Inicialmente se definen los estadígrafos descriptivos de la variable, entre ellos, varianza, desviación estándar, media, entre otros, con el fin de aplicar un criterio de limpia de datos o errores que permita rechazar valores anómalos y, de esta forma, disminuir la dispersión de los datos. Algunos de estos criterios pueden ser el criterio de rechazo 2s, gráficos de dispersión, entre otros.

Previo a la aplicación de técnicas paramétricas para establecer los modelos de previsión de velocidad, debe probarse la normalidad de las variables. Generalmente, el primer paso para establecer ecuaciones de regresión es determinar el coeficiente de correlación entre las variables y gráficos de dispersión de las variables correlacionadas. A partir de este punto, se establecen las variables con mayor fuerza de asociación para establecer modelos de regresión univariables o multivariables. El modelo puede tener un ajuste lineal o de cualquier otro tipo que será determinado por el investigador.

En el caso de los análisis de regresión, deben de ser comprobados para que se cumplan los supuestos de esos análisis y la robustez de la asociación de las variables. Un aspecto fundamental que se debe considerar es que, a partir del análisis bibliográfico, se pueden establecer las variables que generalmente presentan mejor fuerza de asociación y los modelos que mejor se ajustan a la variable de respuesta.

\section{Validación de los modelos de desarrollados}

Una vez desarrollados los modelos de perfil de velocidad para cada uno de los casos previamente establecidos, se procede a comprobar si las velocidades estimadas por el modelo se ajustan a las medidas en campo. Para ello existen dos alternativas: la primera alternativa es la selección de forma aleatoria de uno o más sitios que pertenezcan a la población objeto de estudio y que no formen parte de la muestra empleada para crear el modelo. En este caso, se debe proceder a medir in situ las velocidades de operación y comparar las velocidades reales de operación con las velocidades estimadas por el modelo. Si los diferenciales de las velocidades son inferiores al error de la estimación, puede validarse el modelo. La segunda alternativa consiste en verificar la exactitud de las predicciones de velocidad de los modelos propuestos al comparar las mediciones hechas in situ con otros modelos desarrollados para las mismas condiciones de sitio definidas en este estudio.

En caso de aplicar la segunda alternativa para terreno llano en Costa Rica u otro país, en el que existan diferencias entre normativas, debe realizarse una comprobación como la que se muestra en la Tabla 4. Las ecuaciones 1 y 4 no pueden aplicarse para el caso de terreno llano, y las ecuaciones 2 y 3 difieren en los valores de pendientes de rasante. Por lo tanto, de las ecuaciones propuestas por Fitzpatrick et al. (2000) y García et al. (2012) solo podrían ser objeto de comparación los casos 2,3 y del 5 al 10, que son las combinaciones apropiadas a casos de terreno llano. 


\section{Resultados}

A continuación, se muestra los resultados de la aplicación de la metodología empleada para desarrollar modelos propios de previsión de velocidad de operación para el caso de Costa Rica.

Partiendo de la importancia y la representatividad a nivel nacional, se decide desarrollar los perfiles de velocidad para carreteras clasificadas como rurales de dos carriles de circulación en terreno llano y declaradas rutas nacionales. Estas vías mueven los mayores volúmenes de tránsito a nivel nacional y en ellas ocurren buena parte de los accidentes del tránsito que se desean evaluar a partir de estudios de consistencia del trazado.

\section{Análisis de las carreteras del territorio y definición de parámetros fijos por investigar}

La base de datos necesarios para el estudio se genera a partir del análisis de los proyectos de carreteras disponibles en la Escuela de Ingeniería Civil de la Universidad Latina de Costa Rica y de otros obtenidos mediante la colaboración con diferentes compañías privadas y dependencias gubernamentales.

En la Tabla 5 se muestran los tramos de carretera de dos carriles en terreno llano utilizados para desarrollar los modelos de velocidad. De todos los tramos de los cuales se dispone de su diseño geométrico solo los incluidos en la tabla cumplen con los requisitos de parámetros fijos y buen estado de conservación vial. Estos tramos de carreteras tienen velocidades de diseño comprendidas entre $30 \mathrm{~km} / \mathrm{h}$ y $80 \mathrm{~km} / \mathrm{h}$.

Tabla 5. Trazados obtenidos para el desarrollo de la metodología

\begin{tabular}{|c|c|}
\hline Nombre del Trazado & Kilómetros \\
\hline Barranca-Limonal & 52.00 \\
\hline Limonal-Cañas & 10.00 \\
\hline Paquera-Tambor & 20.24 \\
\hline Orotina-Caldera & 76.64 \\
\hline Tucurrique & 2.80 \\
\hline Total & 161.68 \\
\hline
\end{tabular}

\section{Determinación de los sitios y selección de la muestra}

A partir de la población disponible de sitios en terreno llano de carreteras rurales de interés nacional de Costa Rica, en este caso 161.68 km distribuidos en las diferentes regiones y provincias del país, aunado a que su selección es casuística 'por disponibilidad' se considera un criterio que favorece su representatividad. De esta población se obtienen combinaciones de trazado agrupadas en condiciones similares a las del trabajo desarrollado por Fitzpatrick et al. (2000). Cabe señalar que las condiciones geométricas de los modelos internacionales no coinciden exactamente con las condiciones normadas por el manual del SIECA (2011), que considera la pendiente máxima hasta $5 \%$ para rasantes en terreno llano. En la 
Tabla 6 se presenta la agrupación de sitios que logran ser identificados en la población de tramos de carreteras.

Tabla 6. Muestras de sitios por casos de estudio

\begin{tabular}{|c|c|c|c|}
\hline Caso & Combinación de Trazado & Población & Muestra \\
\hline 2 & Curva horizontal sobre pendiente $(-5 \%<i<0 \%)$ & 34 & 25 \\
\hline 3 & Curva horizontal sobre pendiente (0 \% $<i<5 \%)$ & 34 & 25 \\
\hline 7 & Curva horizontal combinada con acuerdo cóncavo & 26 & 20 \\
\hline 8 & $\begin{array}{l}\text { Curva horizontal combinada con acuerdo convexo sin limitación de } \\
\text { visibilidad }\end{array}$ & 11 & 10 \\
\hline 9 & $\begin{array}{l}\text { Curva horizontal combinada con acuerdo convexo y limitación en la } \\
\text { distancia de visibilidad (Kv } \leq 43 \text { m\%) }\end{array}$ & 19 & 16 \\
\hline 10 & Acuerdo Cóncavo en recta & 30 & 23 \\
\hline 11 & $\begin{array}{l}\text { Acuerdo convexo en recta, sin limitación en la distancia de visibilidad } \\
(\mathrm{Kv}>43 \mathrm{~m} \%)\end{array}$ & 20 & 16 \\
\hline 12 & $\begin{array}{l}\text { Acuerdo convexo en recta, con limitación en la distancia de visibilidad } \\
(\mathrm{Kv} \leq 43 \mathrm{~m} \%)\end{array}$ & 22 & 18 \\
\hline
\end{tabular}

\section{Mediciones de velocidad en los sitios seleccionados}

La toma de muestras de velocidad en el terreno se lleva a cabo con mediciones directas a vehículos en movimiento y bajo las condiciones mencionadas en la sección 2. Este proceso se realiza con una pistola láser de marca Stalker y precisión de $\pm 2.5 \mathrm{~km} / \mathrm{h}$.

El cálculo del tamaño de la muestra de velocidad se realizó considerando valores de $\mathrm{S}=8.5 \mathrm{~km} / \mathrm{h}$, nivel de confianza de $95 \%(\mathrm{~K}=1.96)$, un valor de $\mathrm{U}=1.04$ correspondiente al percentil 85 y error $E=6.5 \mathrm{~km} / \mathrm{h}$. El intervalo de error recomendado por Robertson et al. (1994) es de $1.61 \mathrm{~km} / \mathrm{h}$ hasta $8.05 \mathrm{~km} / \mathrm{h}$. En este sentido, otra consideración importante es que la precisión de la pistola láser no debe ser superior al error elegido, con lo que se evita falsear los resultados estadísticos. Con estos datos se obtuvo un tamaño de muestra igual a 10.

Aunque en ninguno de los casos de estudio el tamaño de la muestra es menor que 10, se procede a calcular, mediante la ecuación 2, la desviación estándar y el error, donde se utilizan los mismos valores de $\mathrm{K}=1.96$ y $\mathrm{U}=1.04$, pero los valores de $\mathrm{N}$ se sustituyen con la muestra de cada caso y S se sustituye con los valores de desviación estándar obtenidos de las mediciones en campo. En la Tabla 7 se muestran los resultados obtenidos. Aunque en algunos casos se sobrepasa el valor de desviación estándar y el error asumido de $6.5 \mathrm{~km} / \mathrm{h}$, en ninguno se supera el error máximo establecido por Robertson et al. (1994) de 8.05 km/h (5 mill/h); por lo tanto, se aceptan los tamaños de muestras determinados.

Independientemente de que autores como García-Ramírez y Damián Alverca (2019), García et al. (2012) y Wilches et al. (2020) refieren que el punto más apropiado para la medición de la velocidad operacional en curvas horizontales es el punto medio, se decidió medir en otros puntos con el objetivo de verificar la veracidad de esta afirmación. Esta idea se justifica en que autores como Maji y Tyagi (2018) determinaron que las velocidades en varios lugares dependen de algunas combinaciones de longitud de curva, curvatura y velocidad en el segmento anterior de la carretera; y que otros, como Dai et al. (2017), determinan la influencia de diferentes parámetros como el radio de la curva horizontal y la pendiente de la curva vertical sobre la velocidad. 
Tabla 7. Valores de desviación estándar y error para el número real de muestras

\begin{tabular}{|c|c|c|c|c|c|c|c|}
\hline Caso & $\mathbf{R}^{2}$ & $\mathbf{p}$ & $\mathbf{U}$ & $\mathbf{K}$ & $\mathbf{N}$ & $\mathbf{S}(\mathbf{K m} / \mathbf{h})$ & $\mathbf{E}(\mathbf{K m} / \mathbf{h})$ \\
\hline 2 & 0.573 & 0.000 & 1.04 & 1.96 & 25 & 9.16 & 4.46 \\
\hline 3 & 0.537 & 0.000 & 1.04 & 1.96 & 25 & 12.06 & 5.87 \\
\hline 7 & 0.629 & 0.000 & 1.04 & 1.96 & 20 & 9.21 & 5.01 \\
\hline 8 & 0.984 & 0.000 & 1.04 & 1.96 & 10 & 3.43 & 2.64 \\
\hline 9 & 0.521 & 0.002 & 1.04 & 1.96 & 16 & 7.61 & 4.63 \\
\hline 10 & 0.119 & 0.106 & 1.04 & 1.96 & 23 & 15.83 & 8.03 \\
\hline 11 & 0.514 & 0.009 & 1.04 & 1.96 & 16 & 8.52 & 5.18 \\
\hline 12 & 0.132 & 0.364 & 1.04 & 1.96 & 18 & 12.16 & 6.97 \\
\hline
\end{tabular}

Por las razones antes comentadas, en curvas horizontales con rasante en inclinación o pendiente se miden velocidades en tres puntos: punto de inicio (PC), punto medio (PM) y punto de final (PT). Con el objetivo de verificar el efecto de la inclinación o la pendiente en la variación de la velocidad de operación, se hizo una prueba t para las muestras tomadas en estos puntos. El análisis estadístico de las mediciones en los puntos mencionados se muestran en la Tabla 8.

Tabla 8. Prueba t para muestras relacionadas de las velocidades medidas en PC, PM y PT

\begin{tabular}{|c|c|c|c|c|c|c|c|c|}
\hline \multirow{3}{*}{$\begin{array}{l}\text { Punto de } \\
\text { medición }\end{array}$} & \multicolumn{5}{|c|}{ Diferencias emparejadas } & \multirow{3}{*}{$\mathrm{t}$} & \multirow{3}{*}{ ggl } & \multirow{3}{*}{$\begin{array}{l}\text { Sig. } \\
\text { (bilateral) }\end{array}$} \\
\hline & \multirow[t]{2}{*}{ Media } & \multirow{2}{*}{$\begin{array}{c}\text { Des- } \\
\text { viación } \\
\text { estándar }\end{array}$} & \multirow{2}{*}{$\begin{array}{l}\text { Media } \\
\text { de error } \\
\text { estándar }\end{array}$} & \multicolumn{2}{|c|}{$\begin{array}{c}95 \% \text { de intervalo de } \\
\text { confianza de la diferencia }\end{array}$} & & & \\
\hline & & & & Inferior & Superior & & & \\
\hline PC-PM & -10.00 & 4.26 & 1.90 & -15.29 & -4.71 & -5.25 & 4 & 0.006 \\
\hline PM-PT & 9.54 & 1.67 & 0.75 & 7.47 & 11.61 & 12.80 & 4 & 0.000 \\
\hline PC-PT & -0.46 & 5.30 & 2.37 & -7.04 & 6.12 & 0.194 & 4 & 0.856 \\
\hline
\end{tabular}

Nota: Minitab

Al comparar las velocidades aplicando la prueba t para muestras relacionadas, se determina que existen diferencias significativas entre los valores de velocidad medidos entre el PC y el PT; sin embargo, estas diferencias de velocidad no son significativas al comparar el PC con el PM y el PM con el PT. Por lo tanto, la velocidad medida en el PM, se puede considerar como la velocidad promedio que desarrolla el vehículo en la curva y, en este sentido, la más apropiada para este tipo de estudios.

\section{Desarrollo de los modelos de predicción del perfil de velocidad}

Los modelos de predicción del perfil de velocidad de operación para carreteras rurales de dos carriles se obtienen con la aplicación de técnicas de regresión. Un aspecto relevante es que los modelos se desarrollan a partir de las variables que muestran mayor fuerza de asociación y no necesariamente a partir de las mismas variables encontradas en otros modelos de la literatura. El procesamiento estadístico de las variables analizadas se elabora con el paquete estadístico Minitab versión 14.12.0. En la Tabla 9 se muestran los resultados estadísticos para los modelos desarrollados. El criterio para la selección de los modelos más apropiados se basa en el análisis 
conjunto del coeficiente de correlación de Pearson (R), el coeficiente de determinación $\left(R^{2}\right)$, el nivel de significación de la ecuación de regresión (p), el error estándar en la estimación del modelo (S) y el estadístico Durbin-Watson (d).

Tabla 9. Resumen de pruebas estadísticas para los modelos de cada caso

\begin{tabular}{|c|c|c|c|c|c|}
\hline Caso & $\mathbf{R}$ & $\mathbf{R}^{2}$ & $\mathbf{p}$ & $\mathbf{S}(\mathbf{k m} / \mathbf{h})$ & $\mathbf{d}$ \\
\hline 2 & 0.757 & 0.573 & 0.000 & 9.16 & 1.363 \\
\hline 3 & 0.733 & 0.537 & 0.000 & 12.06 & 1.560 \\
\hline 7 & 0.793 & 0.629 & 0.000 & 9.21 & 0.987 \\
\hline 8 & 0.9875 & 0.9840 & 0.000 & 3.43 & 1.424 \\
\hline 9 & 0.722 & 0.521 & 0.002 & 7.61 & 1.990 \\
\hline 10 & 0.345 & 0.119 & 0.106 & 15.83 & 0.987 \\
\hline 11 & 0.717 & 0.514 & 0.009 & 8.52 & 1.205 \\
\hline 12 & 0.363 & 0.132 & 0.346 & 12.16 & 1.408 \\
\hline $\begin{array}{l}\text { R: Coeficiente de Pearson, R2: Coeficiente al cuadrado, p: significancia, S: Error estándar en la estimación, D: } \\
\text { Durbin Wanston }\end{array}$ \\
\hline
\end{tabular}

A partir del análisis estadístico, se establece que las ecuaciones de regresión 10 y 12 no satisfacen la generalidad de los estándares de evaluación de este tipo ecuación de pronóstico. Sin embargo, la mayoría de los modelos de predicción establecidos pueden considerarse apropiados a partir del análisis de conjunto de las técnicas antes citadas. En la Tabla 10 se muestran las ecuaciones de predicción de velocidad para los casos seleccionados que satisfacen los análisis estadísticos mencionados anteriormente.

Tabla 10. Modelos de perfil de velocidad para cada caso

\begin{tabular}{|c|c|}
\hline Caso & Ecuación \\
\hline 2 & $\mathrm{~V}_{85}=54.064+0.029 \mathrm{Lc}-0.183 * \mathrm{GC}$ \\
\hline 3 & $V_{85}=39.056+0.047 \mathrm{Lc}+0.009 R+2.791 G$ \\
\hline 7 & $V_{85}=43.639+0.011 * R+0.029 L c$ \\
\hline 8 & $\frac{V^{\lambda}-1}{\lambda^{*} \mathrm{~g}^{\lambda-1}}=6.31+0.08434 R-0.08079 L c, \quad \lambda=5$ \\
\hline 9 & $V_{85}=44.377+0.13 R$ \\
\hline 11 & $V_{85}=70.238-3.260 G+989.523 \mathrm{Kv}^{-1}$ \\
\hline $\begin{array}{l}\text { Nota: LC: longitud de la curva, GC: Grado de curvatura, R: Radio de la curva, l: Coeficiente de la ecuación de regre- } \\
\text { sión, g: Media geométrica de } \mathrm{V}, \mathrm{KV}: \text { tasa de la curva vertical }\end{array}$ \\
\hline
\end{tabular}

\section{Validación de los modelos de velocidad desarrollados}

Los seis modelos propuestos fueron validados comparando sus resultados con velocidades medidas in situ y con velocidades estimadas por modelos de otros autores. En la Tabla 11 se presentan, a modo de ejemplo, los resultados obtenidos para el caso de trazado 8 y su compro- 
bación por las alternativas 1 (comparación con velocidades reales medidas in situ) y alternativa 2 (comparación con otros modelos para casos similares).

Al comparar con los modelos propuestos, estos presentan un excelente ajuste con las velocidades de operación reales medidas in situ; sin embargo, al hacerlo con otros modelos desarrollados para condiciones de otros países como los trabajos de Fitzpatrick et al. (2000) y García et al. (2012) para condiciones similares, se evidencian diferencias importantes. Es importante destacar que el modelo desarrollado por García et al. (2012) se ajusta en mejor medida que el propuesto por Fitzpatrick et al. (2000); aunque ninguno de los dos parece ser apropiado para el caso de Costa Rica.

Tabla 11. Validación de los modelos para caso de trazado 8

\begin{tabular}{|c|c|c|c|c|}
\hline Caso & Ecuación & $\begin{array}{c}\text { Velocidad } \\
\text { Estimada } \\
(\mathrm{km} / \mathrm{h})\end{array}$ & $\begin{array}{c}\text { Velocidad } \\
\text { Medida } \\
(\mathrm{km} / \mathrm{h})\end{array}$ & $\begin{array}{l}\text { Diferencia } \\
(\mathrm{km} / \mathrm{h})\end{array}$ \\
\hline 8 & $\frac{V^{\lambda}-1}{\lambda * g^{\lambda-1}}=6.31+0.08434 R-0.08079 L c$ & 41.98 & \multirow{4}{*}{43.42} & \pm 1.44 \\
\hline \multirow{2}{*}{$8(\mathrm{~F})$} & $V_{85}=105.98-\frac{3709.90}{R}$ & 100.33 & & \pm 56.91 \\
\hline & $V_{85}=104.82-\frac{3574.51}{R}$ & 99.37 & & \pm 55.95 \\
\hline $8(G, D$ y $D)$ & $V_{\text {op }}=79.883-1744.898 / R$ & 77.23 & & \pm 33.81 \\
\hline
\end{tabular}

Nota: Caso 8-A = Modelo desarrollado en este trabajo de investigación, Caso 8-A (F) = Modelo desarrollado por (Fitzpatrick et al., 2000), Caso 8-A (G, D y D) = Modelo desarrollado por García et al. (2012)

La validación de los modelos de predicción propuestos para el caso de Costa Rica mostrados en la Tabla 11 confirma la necesidad de desarrollar modelos propios de velocidad de operación por país o región, si se desea evaluar la seguridad vial a partir de los análisis de consistencia del trazado.

Los modelos de predicción de perfiles de velocidad desarrollados para el caso de Costa Rica constituyen un primer momento de un proyecto encaminado a evaluar la seguridad vial de carreteras a partir del estudio de consistencia del trazado. Es imprescindible aclarar que los modelos propuestos son válidos para carreteras rurales de dos carriles de interés nacional en terreno llano con velocidades de diseño de entre $30 \mathrm{~km} / \mathrm{h}$ y $80 \mathrm{~km} / \mathrm{h}$. El estudio citado permitiría proponer medidas correctivas que pueden incluir mejoras parciales de los trazados existentes, dada la necesidad de disminuir los altos índices de accidentalidad del país.

\section{Conclusiones y recomendaciones}

Se establecen por primera vez para Costa Rica seis modelos de predicción de la velocidad de operación para carreteras rurales de interés nacional en terreno llano.

Los modelos de predicción se desarrollan a partir de una propuesta metodológica propia que puede ser aplicable a otros países con características similares. 
Se constata estadísticamente, durante el procedimiento experimental, que las mediciones de velocidad se deben hacer en el punto medio de la curva horizontal, ya que en este se presenta la velocidad máxima o mínima según el efecto de la pendiente del terreno.

Los seis modelos de predicción de velocidad desarrollados para carreteras rurales de dos carriles denominadas vías nacionales en terreno llano son validados con mediciones de velocidad in situ y comparados con modelos de Fitzpatrick et al. (2000) y García et al. (2012). La comparación de las velocidades medidas in situ con las estimadas por los modelos propuestos está siempre dentro del margen del error de predicción, con lo cual se ratifica su validez.

Se demuestra la necesidad de desarrollar modelos propios de predicción de velocidad de operación, al comparar estos modelos con sus equivalentes disponibles en la literatura internacional.

Una vez desarrollados los modelos para el caso de carreteras nacionales de características rurales en terreno llano, es posible evaluar la consistencia del trazado en proyectos nuevos o existentes en Costa Rica.

\section{Referencias}

Abebe, M. T., \& Belayneh, M. Z. (2018). Identifying and Ranking Dangerous Road Segments a Case of Hawassa-Shashemene-Bulbula Two-Lane Two-Way Rural Highway, Ethiopia. Journal of Transportation Technologies, 8(03), 151-174. https://doi.org/10.4236/jtts.2018.83009

Almeida, R., Vasconcelos, L., \& Bastos Silva, A. (2018). Design Consistency Index for Two-lane Roads Based on Continuous Speed Profiles. PROMET. Traffic\&Transportation, 30(2), 231-239. https:// doi.org/10.7307/ptt.v30i2.2573

Choudhari, T., \& Maji, A. (2019). Effect of Horizontal Curve Geometry on the Maximum Speed Reduction: A Driving Simulator-Based Study. Transportation in Developing Economies, 5(2), 1-8. https://doi. org/10.1007/s40890-019-0082-8

COSEVI (Consejo de Seguridad Vial). (2020). Accidentes. https://datosabiertos.csv.go.cr/dashboards/19683/accidentes/

Dai, Y., Lyu, N., \& Hu, Y. (2017, 8-10, August). Truck Speed Characteristics Analysis of Typical Highway Segments Based on GPS Data. $20174^{\text {th }}$ International Conference on Transportation Information and Safety (ICTIS). Banff, AB, Canada. https://doi.org/10.1109/ICTIS.2017.8047817

Echaveguren, T., Henríquez, C., \& Jiménez-Ramos, G. (2020). Longitudinal Acceleration Models for Horizontal Reverse Curves of Two-Lane Rural Roads. The Baltic Journal of Road and Bridge Engineering, 15(1), 103-125. https://doi.org/10.7250/bjrbe.2020-15.463

Fitzpatrick, K., Wooldridge, M. D., Tsimhoni, O., Collins, J. M., Green, P., Bauer, K. M., Parma, K. D., Koppa, R., Harwood, D. W., Anderson, I., Krammes, R. A., \& Poggioli, B. (2000). Alternative Design Consistency Rating Methods for Two-Lane Rural Highways. U. S. Department of Transportation, Final Report, FHWA-RD-99-172. https://trid.trb.org/view/691718

García-Ramírez, Y. D., \& Alverca, F. (2019). Calibración de ecuaciones de velocidades de operación en carreteras rurales montañosas de dos carriles: Caso de estudio ecuatoriano. Revista Politécnica, 43(2), 37-44. https://doi.org/10.33333/rp.vol43n2.1012

García-Ramírez, Y., Zárate, B., Segarra, S., \& González, J. (2017). Variación diaria y horaria de la velocidad de operación en carreteras rurales de dos carriles en el cantón Loja. Revista Politécnica, 40(1), 45-51. https://doi.org/10.33333/rp.v40i1.864

García, R. A., Delgado, D. E., \& Díaz, E. E. (2012). Modelos de perfil de velocidad para evaluación de consistencia del trazado en carreteras de la provincia de Villa Clara, Cuba. Revista Ingeniería de Construcción, 27(2), 71-82. https://doi.org/10.4067/S0718-50732012000200005 
Goralzik, A., \& Vollrath, M. (2017). The Effects of Road, Driver, and Passenger Presence on Drivers' Choice of Speed: A Driving Simulator Study. Transportation Research Procedia, 25, 2061-2075. https:// doi.org/10.1016/j.trpro.2017.05.400

Kiran, B. N., Kumaraswamy, N., \& Sashidhar, C. (2017). A Review of Road Crash Prediction Models for Developed Countries. American Journal of Traffic and Transportation Engineering, 2(2), 10-25. https://doi.org/10.11648/j.ajtte.20170202.11

Leisch, J. E., \& Leisch, J. P. (1977). New Concepts in Design-Speed Application. Transportation Research Record, 631, 4-14. https://trid.trb.org/view/71966

Li, L., Gayah, V. V., \& Donnell, E. T. (2017). Development of Regionalized SPFs for Two-Lane Rural Roads in Pennsylvania. Accident Analysis \& Prevention, 108, 343-353. https://doi.org/10.1016/j. aap.2017.08.035

Llopis-Castelló, D., Bella, F., Camacho-Torregrosa, F. J., \& García, A. (2018). New Consistency Model Based on Inertial Operating Speed Profiles for Road Safety Evaluation. Journal of Transportation Engineering, Part A: Systems, 144(4), 04018006. https://doi.org/10.1061/jtepbs.0000126

Llopis-Castelló, D., Camacho-Torregrosa, F. J., \& García, A. (2018). Calibration of the Inertial Consistency Index to Assess Road Safety on Horizontal Curves of Two-Lane Rural Roads. Accident. Analysis \& Prevention, 118, 1-10. https://doi.org/10.1016/j.aap.2018.05.014

Llopis-Castelló, D., Findley, D. J., Camacho-Torregrosa, F. J., \& García, A. (2019). Calibration of Inertial Consistency Models on North Carolina Two-Lane Rural Roads. Accident. Analysis and Prevention, 127, 236-245. https://doi.org/10.1016/j.aap.2019.03.013

Llopis-Castelló, D., Findley, D. J., \& García, A. (2020). Comparison of the Highway Safety Manual Predictive Method with Safety Performance Functions Based on Geometric Design Consistency. Journal of Transportation Safety \& Security, 1-22. https://doi.org/10.1080/19439962.2020.1738612

Llopis-Castelló, D., González-Hernández, B., Pérez-Zuriaga, A. M., \& García, A. (2018). Speed Prediction Models for Trucks on Horizontal Curves of Two-Lane Rural Roads. Transportation Research Record: Journal of the Transportation Research Board, 2672(17), 72-82. https://doi. org/10.1177/0361198118776111

Maji, A., Sil, G., \& Tyagi, A. (2018). 85 $5^{\text {th }}$ and $98^{\text {th }}$ Percentile Speed Prediction Models of Car, Light, and Heavy Commercial Vehicles for Four-Lane Divided Rural Highways. Journal of Transportation Engineering, Part A: Systems, 144(5), 04018009. https://doi.org/10.1061/JTEPBS.0000136

Maji, A., \& Tyagi, A. (2018). Speed Prediction Models for Car and Sports Utility Vehicle at Locations Along Four-Lane Median Divided Horizontal Curves. Journal of Modern Transportation, 26(4), 278-284. https://doi.org/10.1007/s40534-018-0162-1

Malaghan, V., Pawar, D. S., \& Dia, H. (2020). Modeling Operating Speed Using Continuous Speed Profiles on Two-Lane Rural Highways in India. Journal of Transportation Engineering, Part A: Systems, 146(11), 04020124. https://doi.org/10.1061/jtepbs.0000447

Robertson, D. H., Hummer, J. E., \& Nelson, D. C. (1994). Spot Speed Studies. In Manual of Transportation Engineering Studies. Institute of Transportation Engineers.

Saleem, T., \& Persaud, B. (2017). Another Look at The Safety Effects of Horizontal Curvature on Rural TwoLane Highways. Accident Analysis and Prevention, 106, 149-159. https://doi.org/10.1016/j. aap.2017.04.001

SIECA, S. de I. E. C. (2011). Manual centroamericano de normas para el diseño geométrico de carrete-

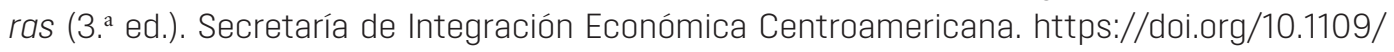
ICIP.2003.1246782

Sil, G., Maji, A., Nama, S., \& Maurya, A. K. (2019). Operating Speed Prediction Model as a Tool for Consistency Based Geometric Design of Four-Lane Divided Highways. Transport, 34(4), 425-436. https:// doi.org/10.3846/transport.2019.10715

Sil, G., Nama, S., Maji, A., \& Maurya, A. K. (2018, January 7-11). The 85 del for Four-Lane Divided Highways in Ideal Free Flow Condition [Paper], 18-23. Transporta- 
tion Research Board 97th Annual Meeting, Washington DC, United States. https://trid.trb.org/ view/1495476

Wilches, F. J., Burbano, J. L. A., \& Sierra, E. E. C. (2020). Vehicle Operating Speeds in Southwestern Colombia: An Important Database for The Future Implementation of Optimization Models for Geometric Design of Roads in Mountain Topography. Data in Brief, 32, 106210. https://doi.org/10.1016/j. dib.2020.106210

Xu, J., Lin, W., \& Shao, Y. (2017). New Design Method for Horizontal Alignment of Complex Mountain Highways Based on "Trajectory-Speed" Collaborative Decision. Advances in Mechanical Engineering, 9(4), 1-18. https://doi.org/10.1177/1687814017695437

Xu, J., Lin, W., Wang, X., \& Shao, Y. M. (2017). Acceleration and Deceleration Calibration of Operating Speed Prediction Models for Two-Lane Mountain Highways. Journal of Transportation Engineering, 143(7), 1-13. https://doi.org/10.1061/JTEPBS.0000050 